\title{
Evidence for a fractionally quantized Hall state with anisotropic longitudinal transport
}

\author{
Jing Xia ${ }^{1 \star \dagger}$, J. P. Eisenstein ${ }^{1}$, L. N. Pfeiffer ${ }^{2}$ and K. W. West ${ }^{2}$
}

At high magnetic fields, where the Fermi level lies in the $\mathbf{N}=\mathbf{0}$ lowest Landau level (LL), a clean two-dimensional electron system (2DES) shows numerous incompressible liquid phases which exhibit the fractional quantum Hall effect (FQHE; ref. 1). These liquid phases do not break rotational symmetry, exhibiting resistivities which are isotropic in the plane. In contrast, at lower fields, when the Fermi level lies in the $N \geq 2$ third and several higher LLs, the 2DES exhibits a distinctly different class of collective states. In particular, near half-filling of these high LLs the 2DES exhibits a strongly anisotropic longitudinal resistance at low temperatures ${ }^{2,3}$. These 'stripe' phases, which do not exhibit the quantized Hall effect, resemble nematic liquid crystals, possessing broken rotational symmetry and orientational order ${ }^{4-8}$. Here we report a surprising new observation: an electronic configuration in the $N=1 \mathrm{LL}$, the resistivity tensor of which simultaneously exhibits a robust fractionally quantized Hall plateau and a strongly anisotropic longitudinal resistance resembling that of the stripe phases.

The sample (details described in Methods) we employ is a square shaped GaAs/AlGaAs heterostructure with edges parallel to the $\langle 110\rangle$ and $\langle 1 \overline{1} 0\rangle$ crystal directions, henceforth referred to as the $\hat{x}$ and $\hat{y}$ directions, respectively. Substantial in-plane magnetic fields $B_{\|}$may be added to the field $B_{\perp}$ perpendicular to the $2 \mathrm{DES}$ plane by tilting the sample at low temperatures. For the present studies, $B_{\|}$lies along the $\hat{x}$, or $\langle 110\rangle$, direction. Figure 1 shows the longitudinal and Hall resistances at $T \approx 15 \mathrm{mK}$ with the magnetic field perpendicular to the 2DES plane (tilt angle $\theta=0$ ). For the field range shown, the Fermi level lies in the lower spin branch of the $N=1 \mathrm{LL}$ where the filling fraction $v \equiv n h / e B_{\perp}$ runs from $v=2$ to $v=3$. Deep minima in the longitudinal resistances and associated plateaux in $R_{x y}$ and $R_{y x}$ clearly signal the presence of FQHE states at $v=7 / 3,5 / 2$ and $8 / 3$. Whereas the $v=7 / 3=2+1 / 3$ and $8 / 3=2+2 / 3$ states may be kin to the well-known $v=1 / 3$ and $2 / 3$ FQHE states in the $N=0$ lowest LL (ref. 1; alternatives do exist; see ref. 9), the $v=5 / 2$ state $^{10}$ is thought to be an example of the non-abelian Moore-Read paired composite fermion state $^{11}$. In addition to these and a few weaker FQHE states, the four known re-entrant integer quantized Hall states ${ }^{12}$, in varying stages of development, are also evident in Fig. 1. These insulating phases are poorly understood but may be related to the 'bubble' phases found in the flanks of the $N \geq 2$ LLs and which also exhibit re-entrant integer Hall quantization ${ }^{2-6}$. As the data in Fig. 1 make clear, the longitudinal and Hall resistances are very similar for current flow along $\langle 110\rangle$ and $\langle 1 \overline{1} 0\rangle$. (The small differences between $R_{x x}$ and $R_{y y}$ for $2.85 \lesssim B_{\perp} \lesssim 3 \mathrm{~T}$ are probably due to extrinsic sample-dependent effects of no relevance here.) Unlike the situation in the $N \geq 2$ LLs, no anisotropic phases have been
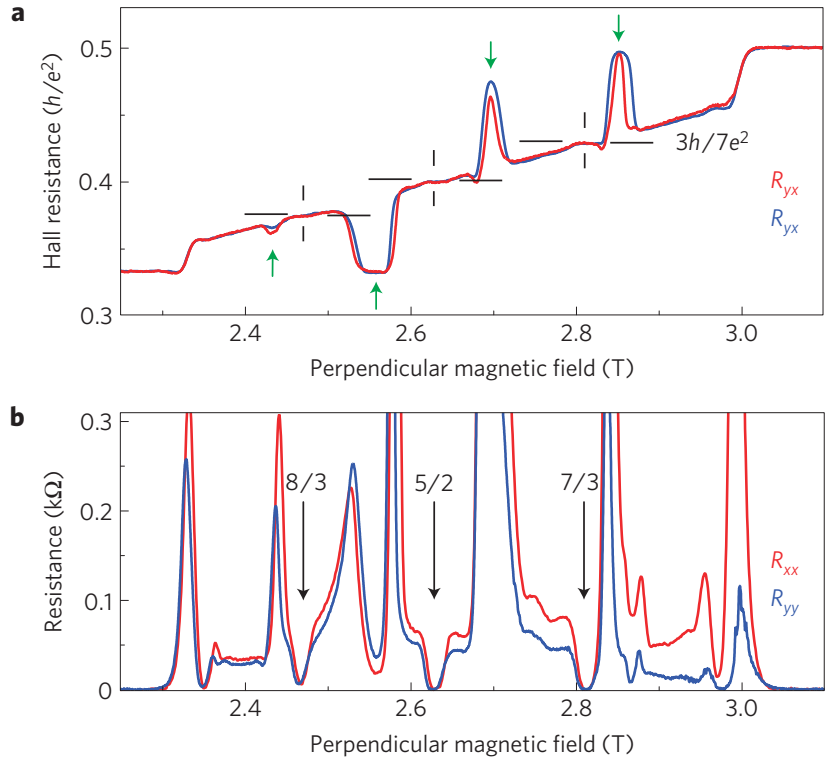

Figure 1 | Hall and longitudinal resistances at $T \approx 15 \mathrm{mK}$ versus magnetic field in the $\boldsymbol{N}=1$ Landau level. $v=7 / 3,5 / 2$, and $8 / 3 \mathrm{FQHE}$ states are indicated by cross-hairs in $\mathbf{a}$ and arrows in $\mathbf{b} . R_{x y}$ and $R_{x x}$ are the Hall and longitudinal resistances, respectively, for mean current flow along the $\langle 110\rangle$ direction; for $R_{y x}$ and $R_{y y}$ mean current flow is along $\langle 1 \overline{1} 0\rangle$. Sample is perpendicular to the magnetic field $\left(\theta=0^{\circ}\right)$. Green arrows in a indicate locations of re-entrant integer quantized Hall states.

found in $2 \mathrm{D}$ electron systems in the $N=1 \mathrm{LL}$, at least in the absence of an external symmetry breaking field such as an in-plane magnetic field $B_{\| \cdot}$. (Anisotropy in the $N=1 \mathrm{LL}$ has been observed in 2D hole systems ${ }^{13,14}$.)

Tilting the 2DES relative to the magnetic field direction has a profound influence on the various collective phases found in the $N=1 \mathrm{LL}$. In agreement with prior studies ${ }^{12,15-17}$, we find that the $v=5 / 2$ FQHE state and the re-entrant integer quantized Hall states are suppressed by an in-plane magnetic field component, $B_{\|}$. In addition to the destruction of these quantized Hall states, the general trend of the longitudinal resistance throughout the $N=1 \mathrm{LL}$ is to become increasingly anisotropic as $B_{\|}$is initially applied, with $R_{x x}$ (for which the mean current direction lies along $B_{\|}$) growing significantly larger than $R_{y y}$ (refs 18,19).

Figure 2 shows the temperature dependence of the longitudinal resistances $R_{x x}$ and $R_{y y}$ at $v=7 / 3$ for various tilt angles $\theta$. As expected, at $\theta=0$ (Fig. 2a) we find $R_{x x}$ and $R_{y y}$ are very nearly equal at all temperatures. Below about $T=100 \mathrm{mK}$ the $v=7 / 3$

\footnotetext{
${ }^{1}$ Condensed Matter Physics, California Institute of Technology, Pasadena, California 91125, USA, ${ }^{2}$ Department of Electrical Engineering, Princeton University, Princeton, New Jersey 08544, USA. †Present address: Department of Physics and Astronomy, University of California, Irvine, California 92697 , USA. *e-mail:xia.jing@uci.edu.
} 

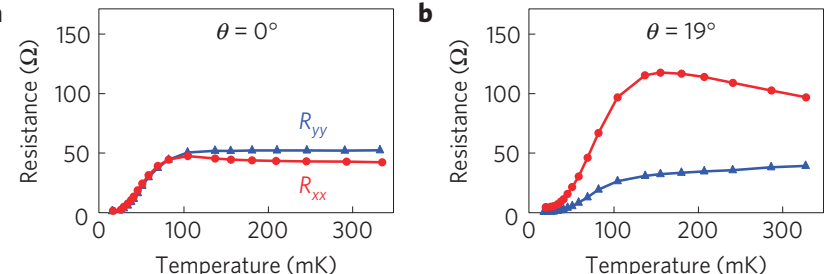

c

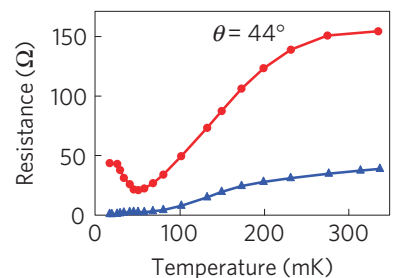

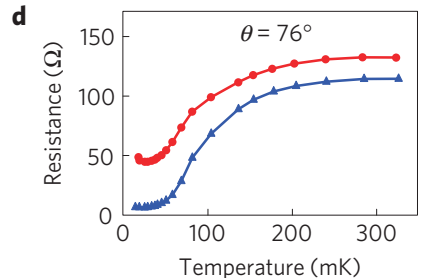

Figure $2 \mid R_{x x}$ and $R_{y y}$ versus temperature at $v=7 / 3$ for $\theta=0^{\circ}, 19^{\circ}, 44^{\circ}$ and $76^{\circ} . R_{x x}$ is shown as red dots and $R_{y y}$ as blue triangles.

FQHE begins to develop, with $R_{x x}$ and $R_{y y}$ dropping rapidly towards zero in unison as the temperature falls. This temperature dependence is well-approximated by simple thermal activation, $R \sim \exp (-\Delta / 2 T)$, with $\Delta \approx 225 \mathrm{mK}$.

Tilting the sample to just $\theta=19^{\circ}$ (Fig. 2b), where $B_{\|}=0.97 \mathrm{~T}$, creates a substantial anisotropy in the longitudinal resistance, with $R_{x x}$ exceeding $R_{y y}$. The anisotropy is present at relatively high temperatures $(T \sim 300 \mathrm{mK}$ ), well above where both resistances begin to fall sharply as the FQHE develops. Increasing the tilt angle to $\theta=44^{\circ}$ (Fig. $2 \mathrm{c}$ ), where $B_{\|}=2.72 \mathrm{~T}$, enhances both the anisotropy and the temperature below which the resistances begin their FQHE-induced fall. This latter effect reflects the tilt-induced increase of the $v=7 / 3$ FQHE energy gap noted previously in similar samples ${ }^{20,21}$. At the large tilt angle of $\theta=76^{\circ}$ (Fig. 2d), the anisotropy in the longitudinal resistance has subsided significantly at high temperatures, in spite of the large in-plane magnetic field $\left(B_{\|}=11.3 \mathrm{~T}\right)$ which breaks rotational symmetry. This surprising return towards isotropic transport has been noted previously and related to mixing of the Landau levels emanating from the two lowest subbands of the confinement potential ${ }^{21}$.

The $\theta=44^{\circ}$ data in Fig. 2c show a curious effect: after falling steadily from $T \approx 250 \mathrm{mK}$ down to about $50 \mathrm{mK}, R_{x x}$, the resistance in the $\langle 110\rangle$ direction, suddenly begins to rise as the temperature is lowered further. No such anomaly is observed in $R_{y y}$, the resistance in the $\langle 1 \overline{1} 0\rangle$ direction, which drops to very small values in the low-temperature limit. Although Fig. 2d demonstrates that this peculiar behaviour has almost vanished by $\theta=76^{\circ}$, Fig. 3a shows it to be quite pronounced at $\theta=66^{\circ}$, where $B_{\|}=6.33 \mathrm{~T}$ at $\nu=7 / 3$.

The $\theta=66^{\circ}$ data in Fig. 3 show three distinct regimes of resistive anisotropy. In the high-temperature regime $(350>T>$ $250 \mathrm{mK}$ ) the resistances $R_{x x}$ and $R_{y y}$ are only weakly temperaturedependent, with $R_{x x}$ exceeding $R_{y y}$ by about a factor of three. As Fig. $3 \mathrm{e}$ shows, the Hall resistances $R_{x y}$ and $R_{y x}$ do not exhibit a quantized Hall plateau in this regime. In the intermediate temperature range $50<T<250 \mathrm{mK}$, the FQHE is beginning to dominate the transport. Both $R_{x x}$ and $R_{y y}$ fall with decreasing temperature and exhibit local minima versus magnetic field at $v=7 / 3$ (see the Supplementary Information). As Fig. 3d illustrates, a Hall plateau at $R_{x y}=R_{y x}=3 h / 7 e^{2}$ appears below about $150 \mathrm{mK}$. The resistive anisotropy remains, with the ratio $R_{x x} / R_{y y}$ growing as the temperature falls. (This behaviour is inconsistent with simple thermal activation; see the Supplementary Information.) Finally, there is the low-temperature regime, demarcated by a sudden change in the sign of $\mathrm{d} R_{x x} / \mathrm{d} T$ at $T \approx 50 \mathrm{mK}$. Below this temperature $R_{x x}$ rises steadily, ultimately reaching $R_{x x} \approx 150 \Omega$ at $T \approx 15 \mathrm{mK}$, our lowest measurement temperature. Throughout

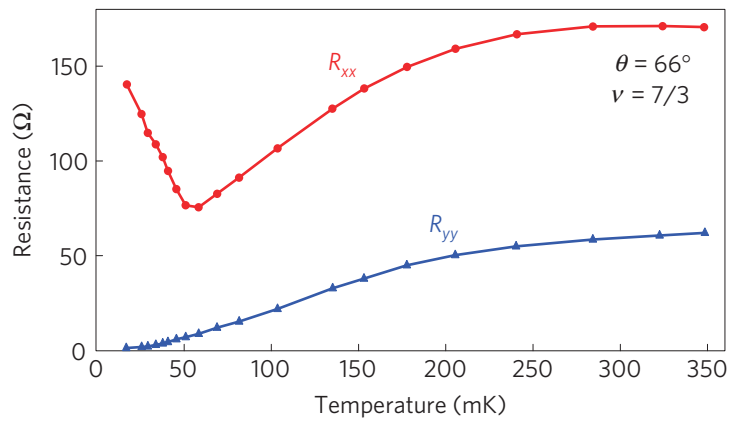

b

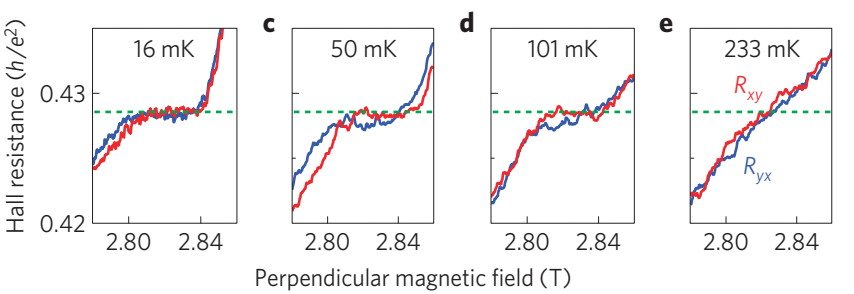

Figure 3 | Hall and longitudinal resistances at $v=7 / 3$ for $\theta=66^{\circ}$.

a, $R_{x x}$ and $R_{y y}$ versus temperature. b-e, Hall resistances $R_{x y}$ and $R_{y x}$ versus magnetic field at various temperatures. Green dashed line indicates the expected location of $v=7 / 3$ Hall plateau.

this low-temperature range the Hall resistances $R_{x y}$ and $R_{y x}$ show well-quantized Hall plateaux, as Fig. 3b,c proves. Intriguingly, $R_{y y}$ continues to fall towards zero in this low-temperature regime, passing smoothly through $T \approx 50 \mathrm{mK}$ with no sign of the abrupt behaviour exhibited by $R_{x x}$.

Consistent with prior work $^{18,19,22}$, when $B_{\|}$is directed along $\langle 1 \overline{1} 0\rangle$ instead of $\langle 110\rangle$, the 'hard' and 'easy' axes of the lowtemperature resistive anisotropy at $7 / 3$ switch to $\langle 1 \overline{1} 0\rangle$ and $\langle 110\rangle$, respectively. Although the anisotropy is reduced in magnitude, the same curious upturn in the hard-axis resistance (now $R_{y y}$ ) is observed at very low temperatures. At $v=8 / 3$ we find that $B_{\|}$induces a similarly strong resistive anisotropy without destroying the quantized Hall plateau, although no clear upturn in the hardaxis resistance has so far been seen.

The resistive anisotropy for $T \gtrsim 250 \mathrm{mK}$ shown in Fig. 3 recalls that observed in the stripe phases of the $N \geq 2$ LLs under similar tilted field conditions. Previous experiments on the $v=9 / 2$ state have demonstrated that a weakly temperature-dependent resistive anisotropy extends to high temperatures $(T \sim 500 \mathrm{mK})$ when a strong in-plane magnetic field is present ${ }^{22}$. Furthermore, in common with the $v=7 / 3$ anisotropy reported here, the orientation of this anisotropy is dictated by the direction of the in-plane magnetic field, with the high-resistance direction usually parallel to $B_{\|}$(refs $\left.18-21,23,24\right)$. We therefore speculate that in the present highly tilted $v=7 / 3$ case, some form of stripe-like density modulation is present at relatively high temperatures and is responsible for the observed anisotropy in the longitudinal resistance. As the temperature is reduced, the FQHE begins to develop and compete with this stripe-like order. As the resistive anisotropy persists to considerably higher temperatures than the FQHE, the energy scale for the stripe-like state exceeds that of the FQHE state. As a result, the quantum Hall fluid, which at these relatively high temperatures remains compressible, will accommodate itself to the density modulation. Unless the modulation is too strong, we believe that it will simply be translated into a spatial modulation of the density of fractionally charged quasiparticles above the FQHE gap. The longitudinal resistance in such a weakly density-modulated FQHE state will certainly be anisotropic at finite temperatures and a quantized Hall plateau can be expected. We note stripes only appear in the $N=1 \mathrm{LL}$ when a 
tilted field is applied, in contrast to the situation in the $N \geq 2$ LLs. Quantum fluctuations ${ }^{7}$ are much stronger in the $N=1 \mathrm{LL}$ and it seems plausible that they will limit the density modulation in any tilt-induced stripe phase.

Although the above scenario may explain the data in Fig. 3 in the high and intermediate temperature ranges, it does not readily account for the upturn in $R_{x x}$ at $T \approx 50 \mathrm{mK}$ and the persistence of the Hall plateau down to $T \approx 15 \mathrm{mK}$. Indeed, the abruptness of the upturn indicates the emergence of a new electronic configuration in the low-temperature regime. The $v=7 / 3$ longitudinal resistance data shown in Fig. 3a are, for $T \lesssim 50 \mathrm{mK}$, again reminiscent of that seen in the stripe phases at half-filling of the $N \geq 2$ LLs (for example at $v=9 / 2,11 / 2$, and so on), only now as they appear in the absence of an in-plane magnetic field. Under these conditions a strong resistive anisotropy develops only at low temperatures $(T \lesssim 100 \mathrm{mK})$. The resistance becomes large in one crystallographic direction and very small in the orthogonal direction. Here, at $v=7 / 3, R_{x x}$ rises rapidly below $T=50 \mathrm{mK}$, reaching approximately $150 \Omega$ at $T \approx 15 \mathrm{mK}$, whereas $R_{y y}$ concurrently falls to $\sim 1 \Omega$. However, in sharp contrast to the anisotropic phases at $v=9 / 2$, $11 / 2$, and so on, the anisotropic $v=7 / 3$ state exhibits a robustly quantized Hall plateau down to the lowest temperatures studied.

The simultaneous presence, at $v=7 / 3$ in the present instance, of an accurately quantized Hall plateau and strong, highly temperature-dependent anisotropy in the longitudinal resistance, has not been encountered experimentally before. There have however, been theoretical suggestions of fractional quantized Hall phases with broken rotational symmetry. Using a variational approach, Musaelian and Joynt ${ }^{25}$ suggested that in wide quantum wells (which soften the short-range part of the Coulomb interaction) the 2DES at $v=1 / 3$ in the $N=0$ Landau level might be most stable in a phase analogous to a classical nematic liquid crystal. Although exhibiting gapless neutral collective modes of the director order parameter, Musaelian and Joynt nonetheless argue that charged excitations are gapped and thus the system would still exhibit the FQHE. How the transport coefficients would behave as functions of temperature, and what might pin the order parameter, was not addressed. More recently, Mulligan, Nayak and $\mathrm{Kachru}^{26}$ ), using an effective field theory approach, have predicted a transition from an isotropic to an anisotropic FQHE state with nematic order. The transition is driven by subtle changes in the electron-electron interaction which, in the light of the present experimental results, Mulligan, Nayak and Kachru speculated arise from couplings between an in-plane magnetic field and the finite thickness of the 2DES. Although Mulligan, Nayak and Kachru predict that both $R_{x x}$ and $R_{y y}$ will ultimately vanish at $T=0$, they also find a regime, as in our experiments, where one of the two resistances increases as the temperature falls while the other resistance decreases.

In addition to the homogeneous nematic FQHE phases mentioned above, it is also possible that our results reflect a phase-separated 2DES. In analogy with the situation at $v=9 / 2$, a simple stripe phase picture at $v=7 / 3$ (consisting, for example, of alternating stripes of $v=2$ and $v=3$ ) would yield anisotropic longitudinal transport but not quantization of the Hall resistance. (We are ignoring the possible formation of isotropic 'bubble' phases $^{4-6}$ away from half-filling.) Alternatively, one can imagine that at $v=7 / 3$ the electrons in the $1 / 3$-filled $N=1$ Landau level exist in an anisotropic version of the quantized Hall insulator (QHI) first encountered experimentally at $v \lesssim 1 / 3$ in the $N=0 \mathrm{LL}$ in relatively disordered samples ${ }^{27,28}$. In Shimshoni and Auerbach's theory ${ }^{27}$, the QHI is modelled as a collection of incompressible FQHE puddles immersed in an insulating background fluid and connected to one another by tunnel junctions. Shimshoni and Auerbach find that the longitudinal resistance diverges as $T \rightarrow 0$ and, remarkably, the Hall resistance is quantized at the value appropriate to the FQHE puddles. Adjusting this scenario to $v \lesssim 7 / 3=2+1 / 3$ indicates a Hall resistance quantized at $R_{x y}=3 h / 7 e^{2}$ and a longitudinal resistance $R_{x x}$ which, although vanishing at $T=0$, exhibits quasi-insulating behaviour $\left(d R_{x x} / d T<0\right)$ in some intermediate temperature range. By construction, the Shimshoni and Auerbach model is isotropic. Thus, although our $R_{x x}, R_{x y}$, and $R_{y x}$ data are broadly consistent with the Shimshoni and Auerbach model, it does not encompass the behaviour we observe in $R_{y y}$. Nevertheless, it seems at least plausible that, by assuming the FQHE puddles to be oblate and consistently oriented relative to the in-plane magnetic field, anisotropy in the longitudinal resistance would emerge from the model. Whether the different temperature dependences of $R_{x x}$ and $R_{y y}$ can also be accommodated is less clear.

\section{Methods}

The sample we employ is a standard GaAs/AlGaAs heterostructure grown by molecular beam epitaxy. A $40 \mathrm{~nm}$ GaAs quantum well is embedded in thick $\mathrm{Al}_{0.24} \mathrm{Ga}_{0.76}$ As cladding layers. Si doping sheets in each cladding layer create a $2 \mathrm{DES}$ in the lowest subband of the GaAs quantum well. After illumination, the density and mobility of this 2DES are $n=1.6 \times 10^{11} \mathrm{~cm}^{-2}$ and $\mu=16 \times 10^{6} \mathrm{~cm}^{2} \mathrm{Vs}^{-1}$, respectively. The sample is a $5 \mathrm{~mm}$ square chip the edges of which are parallel to the $\langle 110\rangle$ and $\langle 1 \overline{1} 0\rangle$ crystal directions, henceforth referred to as the $\hat{x}$ and $\hat{y}$ directions, respectively. InSn ohmic contacts are positioned at the corners and side midpoints of the sample. Longitudinal resistance $\left(R_{x x}\right.$ and $\left.R_{y y}\right)$ measurements are performed by driving an a.c. current (typically $2 \mathrm{nA}$ at $13 \mathrm{~Hz}$ ) between midpoint contacts on opposite sides of the sample and detecting the resulting voltage difference between corner contacts on one side of the mean current axis. For the Hall resistances ( $R_{x y}$ and $R_{y x}$ ), the voltage difference between the two midpoint contacts on opposite sides of the current axis is recorded. The sample is mounted on a rotating platform (allowing for the application of an in-plane magnetic field component) thermally linked to the mixing chamber of a dilution refrigerator.

Received 18 May 2011; accepted 14 September 2011; published online 23 October 2011

\section{References}

1. Das Sarma, S. \& Pinczuk, A. Perspectives on Quantum Hall Effects (Wiley, 1997).

2. Lilly, M. P., Cooper, K. B., Eisenstein, J. P., Pfeiffer, L. N. \& West, K. W. Evidence for an anisotropic state of two-dimensional electrons in high Landau levels. Phys. Rev. Lett. 82, 394-397 (1999).

3. $\mathrm{Du}, \mathrm{R}$. R. et al. Strongly anisotropic transport in higher two-dimensional Landau levels. Solid State Commun. 109, 389-394 (1999).

4. Koulakov, A. A., Fogler, M. M. \& Shklovskii, B. I. Charge density wave in two-dimensional electron liquid in weak magnetic field. Phys. Rev. Lett. 76, 499-502 (1996).

5. Fogler, M. M., Koulakov, A. A. \& Shklovskii, B. I. Ground state of a two-dimensional electron liquid in a weak magnetic field. Phys. Rev. B 54, 1853-1871 (1996).

6. Moessner, R. \& Chalker, J. T. Exact results for interacting electrons in high Landau levels. Phys. Rev. B 54, 5006-5015 (1996).

7. Fradkin, E. \& Kivelson, S. A. Liquid-crystal phases of quantum Hall systems. Phys. Rev. B 59, 8065-8072 (1999).

8. Fradkin, E., Kivelson, S. A., Lawler, M. J., Eisenstein, J. P. \& Mackenzie, A. P. Nematic Fermi fluids in condensed matter physics. Annu. Rev. Conden. Matter Phys. 1, 153-178 (2010).

9. Read, N. \& Rezayi, E. Beyond paired quantum Hall states: Parafermions and incompressible states in the first excited Landau level. Phys. Rev. B 59, 8084-8092 (1999).

10. Willett, R. et al. Observation of an even-denominator quantum number in the fractional quantum Hall effect. Phys. Rev. Lett. 59, 1776-1779 (1987).

11. Moore, G. \& Read, R. Nonabelions in the fractional quantum Hall effect. Nucl. Phys. B 360, 362-396 (1991).

12. Eisenstein, J. P., Cooper, K. B., Pfeiffer, L. N. \& West, K. W. Insulating and fractional quantum Hall states in the first excited Landau level. Phys. Rev. Lett. 88, 076801 (2002).

13. Shayegan, M., Manoharan, H. C., Papadakis, S. J. \& De Poortere, E. P. Anisotropic transport of two-dimensional holes in high Landau levels. Physica E 6, 40-42 (2000).

14. Manfra, M. J. et al. Impact of spin-orbit coupling on quantum Hall nematic phases. Phys. Rev. Lett. 98, 206804 (2007).

15. Eisenstein, J. P. et al. Collapse of the even-denominator fractional quantum Hall effect in tilted fields. Phys. Rev. Lett. 61, 997-1000 (1988).

16. Eisenstein, J. P., Willett, R. L., Stormer, H. L., Pfeiffer, L. N. \& West, K. W. Activation energies for the even-denominator fractional quantum Hall effect. Surf. Sci. 229, 31-33 (1990). 
17. Csathy, G. et al. Tilt-induced localization and delocalization in the second Landau level. Phys. Rev. Lett. 94, 146801 (2005).

18. Pan, W. et al. Strongly anisotropic electronic transport at Landau level filling factor $v=9 / 2$ and $v=5 / 2$ under a tilted magnetic field. Phys. Rev. Lett. 83, 820-823 (1999).

19. Lilly, M. P., Cooper, K. B., Eisenstein, J. P., Pfeiffer, L. N. \& West, K. W. Anisotropic states of two-dimensional electron systems in high Landau levels: Effect of an in-plane magnetic field. Phys. Rev. Lett. 83, 824-827 (1999).

20. Dean, C. R. et al. Contrasting behavior of the $5 / 2$ and $7 / 3$ fractional quantum Hall effect in a tilted field. Phys. Rev. Lett. 101, 186806 (2008).

21. Xia, J., Cvicek, V., Eisenstein, J. P., Pfeiffer, L. N. \& West, K. W. Tilt-induced anisotropic to isotropic phase transition at $v=5 / 2$. Phys. Rev. Lett. 105, 176807 (2010).

22. Cooper, K. B., Lilly, M. P., Eisenstein, J. P., Pfeiffer, L. N. \& West, K. W. Onset of anisotropic transport of two-dimensional electrons in high Landau levels: Possible isotropic-to-nematic liquid-crystal phase transition. Phys. Rev. B 65, 241313(R) (2002).

23. Jungwirth, T., MacDonald, A. H., Smrcka, L. \& Girvin, S. M. Field-tilt anisotropy energy in quantum Hall stripe states. Phys. Rev. B 60, 15574-15577 (1999)

24. Stanescu, T. D., Martin, I. \& Phillips, P. Finite-temperature density instability at high Landau level occupancy. Phys. Rev. Lett. 84, 1288-1291 (2000).

25. Musaelian, K. \& Joynt, R. Broken rotation symmetry in the fractional quantum Hall system. J. Phys. Cond. Matter. 8, L105-L110 (1996).
26. Mulligan, M., Nayak, C. \& Kachru, S. Isotropic to anisotropic transition in a fractional quantum Hall state. Phys. Rev. B 82, 085102 (2010).

27. Shimshoni, E. \& Auerbach, A. Quantized Hall insulator: Transverse and longitudinal transport. Phys. Rev. B 55, 9817-9823 (1997).

28. Hilke, M. et al. Experimental evidence for a two-dimensional quantized Hall insulator. Nature 395, 675-677 (1998).

\section{Acknowledgements}

We are grateful to C. Nayak and S. Kivelson for useful discussions. This work was supported by Microsoft Project Q. The work at Princeton was partially funded by the Gordon and Betty Moore Foundation as well as the National Science Foundation MRSEC Program through the Princeton Center for Complex Materials (DMR-0819860).

\section{Author contributions}

J.X. and J.P.E. conceived the project. L.N.P. and K.W.W. fabricated the samples. J.X. performed the experiment. J.X. and J.P.E. discussed the data and co-wrote the manuscript.

\section{Additional information}

The authors declare no competing financial interests. Supplementary information accompanies this paper on www.nature.com/naturephysics. Reprints and permissions information is available online at http://www.nature.com/reprints. Correspondence and requests for materials should be addressed to J.X. 